\title{
Clinical predictive factors in prostatic artery embolization for symptomatic benign prostatic hyperplasia: a comprehensive review
}

\author{
Fei Sun, Vanesa Lucas-Cava, Francisco Miguel Sánchez-Margallo \\ Jesus Uson Minimally Invasive Surgery Centre, Caceres, Spain \\ Contributions: (I) Conception and design: F Sun; (II) Administrative support: None; (III) Provision of study materials or patients: None; (IV) \\ Collection and assembly of data: F Sun, V Lucas-Cava; (V) Data analysis and interpretation: F Sun, V Lucas-Cava; (VI) Manuscript writing: All \\ authors; (VII) Final approval of manuscript: All authors. \\ Correspondence to: Fei Sun. Jesus Uson Minimally Invasive Surgery Centre, Carretera N-521, km. 41,8. 10071, Cáceres, Spain. \\ Email: feisun@ccmijesususon.com.
}

\begin{abstract}
Prostatic artery embolization (PAE) has been established as a routine treatment for symptomatic benign prostatic hyperplasia $(\mathrm{BPH})$ all over the world. With increasing clinical experience in the last decade, investigators have sufficient data to assess predictive factors with the purpose to guide patient selection and counseling for PAE or to individualize therapeutic plans after PAE. This paper is a comprehensive review to introduce the concept of clinical predictors and give a systemic classification of various predictive factors in PAE. The authors review each individual factor and its predictive capability and discuss the possible reasons for the inconsistent or conflicting findings in the literature. Based on current evidence, the baseline prostate volume, in particular the transition zone volume and transition zone index; 24 h post-PAE prostate-specific antigen (PSA) level; and prostate infarction and prostate volume reduction at $1-3$ months have potential in prediction of treatment outcomes. Patients with Adenomatous-dominant BPH or with indwelling bladder catheter before PAE may have more benefits from PAE. Baseline intravesical prostatic protrusion (IPP), C-reactive protein (CRP) level at $48 \mathrm{~h}$ and early detection of prostate infarct at 1 day and 1 week after PAE need further investigating.
\end{abstract}

Keywords: Prostatic artery embolization (PAE); benign prostatic hyperplasia (BPH); lower urinary tract symptoms (LUTS); predictive factors

Submitted Jan 14, 2020. Accepted for publication Jun 09, 2020.

doi: $10.21037 /$ tau-20-437

View this article at: http://dx.doi.org/10.21037/tau-20-437

\section{Introduction}

Prostatic artery embolization (PAE) has emerged as a minimally invasive technique in the management of lower urinary tract symptoms (LUTS) related to benign prostatic hyperplasia (BPH) and been widely accepted in interventional radiology community during the last decade. Shortly after the proof of concept study in animal experiments showed the technical safety of PAE $(1,2)$, initial clinical reports demonstrated that PAE was a viable technique with promising short-term outcomes in relief of LUTS $(3,4)$. Since then, PAE has gained in popularity, and a multitude of clinical trials have been conducted with efforts to evaluate medium- and long-term outcomes on clinical efficacy and safety $(5,6)$, optimize the types and sizes of embolic agents $(7,8)$, refine the embolization and imaging techniques (9-11), discuss the ideal patient selection (12-14), and explore the underlying therapeutic mechanisms $(15,16)$. As a result, in an official position statement of Society of Interventional Radiology, PAE for BPH has been indicated as a novel and promising therapy that appears safe and efficacious with high patient satisfaction and low repeat intervention rates (17). More recently, the UK's National Institute for Health and Care Excellence (NICE) has issued a guideline approving PAE as a standard procedure in the management of symptomatic BPH (18). With 
Table 1 Summary of age of patients undergone PAE

\begin{tabular}{lcccc}
\hline $\begin{array}{l}\text { Authors and } \\
\text { References }\end{array}$ & Country & Cases & $\begin{array}{c}\text { Range of } \\
\text { age (years) }\end{array}$ & $\begin{array}{c}\text { Mean age } \\
\text { (years) }\end{array}$ \\
\hline Pisco et al. (6) & Portugal & 630 & $40-89$ & 65.1 \\
Wang et al. (23) & China & 157 & $54-91$ & 69.5 \\
de Assis et al. (24) & Brazil & 93 & $51-86$ & 63.4 \\
Bagla et al. (12) & USA & 78 & $48-81$ & 65.2 \\
Grosso et al. (25) & Italy & 13 & $51-90$ & 75.9 \\
\hline
\end{tabular}

time and more clinical experience in the practice of PAE, investigators have shown increasing interest in assessing potential predictive factors in PAE therapy in order to guide patient selection and improve therapeutic efficacy. The purpose of this review is to summary the favorable clinical variables that have potential to predict the desired outcomes after PAE therapy.

\section{Concept and classification of clinical predictors in PAE}

Clinical predictors refer to biomedical factors known to influence diagnosis or predict health outcomes (19). Clinical research on predictors in PAE is for prognostic prediction to determine possible outcomes after intervention. In comparison to the diagnostic prediction models, the main difference is the time point on evaluation. In diagnosis, predictive factors are evaluated at the same time in prediction of a suspected disease, thus these studies usually involve cross-sectional analyses (20). Since prediction models in PAE estimate the clinical responses to therapy, such as changes in international prostate symptom score (IPSS), quality of life (QoL), and maximal flow rate (Qmax), which occur in a certain period after PAE; prediction in PAE is typically a longitudinal study.

Clinical predictors in PAE are the clinical variables that have significant correlation with outcomes after treatment and have predictive capabilities for clinical prognosis. These variables involve clinical manifestation, pathological features, and imaging and laboratory findings. Although the different embolic agents of specific size as well as PAE-related technical considerations, e.g., bilateral or unilateral embolization, may have correlation with clinical outcomes (21), they are not considered predictors in the clinical setting, where clinical predictors are either primarily applied in selection of the patients who may more likely benefit in the treatment of PAE, or evaluated early after PAE for the probability of some clinical outcomes. According to clinical features and applications, we suggest classifying the predictors into three types:

* Type I predictors: baseline clinical data collected before PAE, including patient demography, clinical signs and symptoms, pathological features, lab tests, etc., for example, age, IPSS score, uroflowmetry data, prostate volume and associated imaging parameter (transition zone index, intravesical prostatic protrusion), etc. Type I predictors serve to optimize patient selection for PAE.

* Type II predictors: acute clinical data obtained from 1 day to 1 week after PAE, for example, 24 h-PSA, C-reactive protein level, prostate ischemia etc., which are used in prediction of short- or mediumterm outcomes.

* Type III predictors: early clinical data obtained 1-3 months after PAE for predicting long-term outcomes, such as magnetic resonance imaging (MRI) findings including prostate infarction and prostate volume reduction.

\section{Current evidence of clinical predictors in PAE}

Age

$\mathrm{BPH}$ is a chronic progressive disease. In Europe, clinical $\mathrm{BPH}$ with moderate-to-severe symptoms was reported in $14 \%$ of men aged $40-49$ years and the proportion doubles with each decade of life (22). According to recent reports of PAE from various countries, the age range was 40-91 years with the range of mean age of $63.4-75.9$ years and the median mean age of 65.2 years (Table 1) $(6,12,23-25)$. A case report showed successful management of PAE in a 97-year-old patient (26), who is so far the oldest patient in the literature. Since most elderly patients with BPH have various comorbidities and many have chronic or permanent administration of antiplatelet agents $(27,28)$, they are not suitable for conventional surgery or general anesthesia to relieve LUTS. By contrast, PAE as a minimally invasive intervention has no such limitations. In a recent report of PAE, 101 out of 157 patients (64.3\%) had been assessed by anesthesiologists and urologists and excluded from surgery owing to cardiac, pulmonary, or other diseases (23). PAE is performed as an outpatient procedure and does not require general anesthesia in clinical practice, showing remarkable advantages in the management of elderly patients with 
Table 2 Correlations among prostate symptom score, prostate volume and Qmax

\begin{tabular}{|c|c|c|c|c|c|c|c|}
\hline $\begin{array}{l}\text { Authors and } \\
\text { References }\end{array}$ & $\begin{array}{c}\text { Study design + } \\
\text { measurement } \\
\text { techniques }\end{array}$ & \multicolumn{2}{|c|}{ IPSS vs. PV } & \multicolumn{2}{|c|}{ Qmax vs. PV } & \multicolumn{2}{|c|}{ IPSS vs. Qmax } \\
\hline Barry et al. (33) & Cli + TAUS & 0.09 & 0.22 & -0.14 & 0.06 & -0.07 & 0.27 \\
\hline Bosch et al. (34) & Cli + TRUS & $\mathrm{N} / \mathrm{A}$ & & -0.05 & 0.33 & $\mathrm{~N} / \mathrm{A}$ & \\
\hline Ezz el Din et al. (37) & Cli + TRUS & 0.03 & $>0.05$ & N/A & & $\mathrm{N} / \mathrm{A}$ & \\
\hline Lepor et al. (38) & Cli + TRUS & 0.13 & $>0.05$ & -0.40 & 0.001 & $\mathrm{~N} / \mathrm{A}$ & \\
\hline Øverland et al. (39) & Com + TRUS & 0.176 & $\mathrm{~N} / \mathrm{A}$ & $\mathrm{N} / \mathrm{A}$ & & -0.278 & N/A \\
\hline
\end{tabular}

Cli, Clinic-based population; Com, Community-based population; TAUS, transabdominal ultrasound; TRUS, transrectal ultrasound.

BPH. However, it also has technical challenges. In elderly patients, arterial arthrosclerosis is common so that the prostatic artery and its intraprostatic branches may present with local stenoses or occlusion, resulting in technical failure or less responsive to PAE (21). Identification of the clinical predictor of age or potential risk factor of age in PAE is of clinical implications. Unfortunately, there are very few reports investigating correlation of the age of patients with the clinical outcomes following PAE.

In a single-center retrospective study, Bilhim et al. (21) used a Cox proportional hazards model with multivariate analysis to test correlation with clinical failure after PAE, and found the older age ( $>65$ years) was the independent predictors of clinical failure (regression coefficient, 0.034; hazard ratio, $1.035 ; 95 \% \mathrm{CI}: 1.013-1.058 ; \mathrm{P}=0.002$ ), indicating that there is an $3.5 \%$ increase in the event of clinical failure relative to a one year increase in patient age. In other words, a patient of 75 years old would have approximately one third more probability (35\%) of clinical failure when compared to a patient of 65 years of age. Therefore, the authors concluded that younger age (up to 65 years) was predictor of better clinical outcome.

By contrast, Maclean et al. (29) assessed the age and others parameters as independent variables and their correlation with clinical improvement at 12 months in 73 patients. The authors did not find significant correlation between age and change in IPSS with Pearson's bivariate analysis $(\mathrm{P}=0.167, \mathrm{r}=-0.151)$ and multiple linear regression $(\mathrm{P}=0.090$, $\mathrm{r}=-0.042)$. Similarly, in a post hoc analysis of 48 patients,
Abt et al., identified a weak but statistically non-significant correlation between age and IPSS changes at 12 weeks after $\mathrm{PAE}(\mathrm{P}=0.11, \mathrm{r}=-0.23)(30)$.

\section{Prostate volume}

Ever since the concept of PAE in the management of symptomatic BPH was introduced in 2008 (1), controversy remains over PAE focusing on the prostate volume and shrinkage of the enlarged prostate size with regard to its technical rationale and clinical implications. When early clinical reports by interventional radiologists attracted attention of urology community, the initial feedback was skeptical with main criticisms of the reliance on imagingbased reduction in prostate volume and naïve prostatocentric concepts of BPH $(31,32)$. Given that PAE exerts its therapeutic effects with a major technical rationale targeting on the mass effect as the pathologic static component of $\mathrm{BPH}$, questions may arise with regard to whether baseline prostate volume is correlated with clinical outcome after PAE and the hypothesis that early reduction of the prostate volume after PAE may be predictive of middle- and longterm clinical outcomes.

Correlations among LUTS, prostate volume and Qmax have long been debated with inconsistent findings in the literature. The discrepancy varies from no correlation, weak, modest through moderate correlations between prostate volume and symptoms or Qmax, and symptoms and Qmax, which is summarized in Table $2(33-41)$. The lack of 
consensus is multifactorial, such as different general sample selection, for example, community based- or clinic-based population; different imaging techniques for measurement of prostate volume including transabdominal ultrasound (TAUS), transrectal ultrasound (TRUS), or MRI; and different specific inclusion or exclusion criteria in sample selection in clinic-based or community based population.

It is not surprising that no strong correlations exist among total prostate volume (TPV), IPSS or American Urological Association (AUA) symptom score, and Qmax in patients with BPH. It can be explained in several aspects. Firstly, IPSS or AUA symptom score is a measure of symptom severity of clinical $\mathrm{BPH}$, and the symptoms involved are not BPH-specific. Instead, the symptoms are associated with various pathological changes in the urinary bladder and/or prostate. Lepor et al. (42) reported that lower urinary tract symptoms are even not gender specific and there was no statistically significant difference in either mean AUA symptom scores or in the percentage of mild, moderate, and severe symptoms between males and females. This may be attributed to the fact that LUTS can arise from primary bladder disorders such as overactive bladder, agerelated detrusor dysfunction, and interstitial cystitis (43). Secondly, even in patients with benign prostatic enlargement (BPE) and bladder outlet obstruction (BOO), dysfunction of the bladder secondary to BOO, especially in late decompensated stage, has to be considered (15). With prolonged obstruction, the bladder mass substantially increases, the detrusor compliance and contractility decrease, resulting in a decompensated state of the bladder that is associated with diminished urinary stream, hesitancy, intermittency, and increased residual urine. These secondary symptoms and associated impaired urinary flow rate at the decompensated stage may not correlate with the TPV. Similarly, even after technically successful transurethral resection of the prostate (TURP), some patients may still present with the secondary LUTS due to decompensation of the urinary bladder. Thirdly, the heterogeneity of the clinical BPH is well known. LUTS/BPH may result from two pathological components, the static and dynamic one. Thus, the dynamic component may be a common reason for LUTS in BPH patients with a relatively small prostate size. Lastly, the location the nodular hyperplasia may play a critical role in producing LUTS. As explained by Barry (33), in some patients a relatively small degree of hyperplasia in the periurethral area might cause considerable physiological obstruction, while in other individuals, considerable hyperplasia can occur without producing obstruction.
Accordingly, investigation on transition zone volume (TZV) and transition zone index (TZI) as likely more sensitive prostate size markers have drawn much attention.

Patients in PAE are a highly selected cohort $(6,13,44)$, in which the enrolled subjects are commonly older than 40 years with moderate-to severe LUTS and suspected BOO or acute urinary retention (AUR). The patients are usually refractory to medical therapy, including failure to $\alpha$-blocker treatment; thus, the patients with BPH symptoms due to the dynamic component are at least excluded in part, leaving more patients with symptoms due to the mass effect. Furthermore, common exclusion criteria in patient selection before PAE include malignancy, large bladder diverticula or stones, neurogenic bladder, detrusor failure, urethral stenosis, etc. $(6,13,44)$. In some studies $(6,29)$, the patients with equivocal or non-BOO identified with urodynamic study were also excluded. This further limits the candidates for PAE to those with LUTS and BOO, more likely resulting from BPE. Therefore, it seems to be reasonable to expect a relatively stronger correlation between prostate volume and clinical symptoms in this group of patients compared with other cohorts based on community or clinical population in the previous literature as discussed above. This is a major premise upon which we hypothesize the baseline TPV might be associated clinical outcomes following PAE.

\section{Baseline TPV}

From anatomic and pathological perspective, a larger prostate usually has larger-caliber target arteries with hypervascular lesions, enabling injection more embolic agents to induce more ischemia and infarct. As a result, the more prostate volume reduction after PAE and can be expected and better clinical outcomes are achieved (13). Although no correlation between prostate volume and prostatic artery diameter was observed in a report of angiographic anatomic study in patients before PAE (45), Wang et al. noted significant difference of prostatic artery diameter $(1.7 \pm 0.5$ vs. $0.9 \pm 0.4 \mathrm{~mm}, \mathrm{P}<0.05)$ between PAE patients with large prostate size $(>80 \mathrm{~mL})$ and with medium prostate size $(50-80 \mathrm{~mL})(13)$.

Current evidence is conflicting on the predicting role of baseline prostate volume for clinical outcomes after PAE. In a single centre study in 86 patients, Maclean et al. (29) tested the relationship between baseline TPV and symptomatic improvement after PAE. Pearson's correlation test showed a modest correlation between baseline TPV and changes in IPSS at 12 months $(r=-0.324, P=0.002)$. The 
results indicated that approximately $10 \%\left(\mathrm{r}^{2}=0.105\right)$ of the variation in clinical improvement in IPSS following PAE can be explained by baseline TPV. Of note, this cohort is only limited to patients with benign prostatic obstruction (BPO) because all patients identified as equivocal or nonobstructive in pre-PAE urodynamic test were excluded in enrolment (29). Similarly, Abt et al. (30) reported 48 patients with moderate to severe LUTS. Spearman rank correlations test showed that relief in IPSS was significantly correlated with baseline TPV $(r=-0.35, \mathrm{P}=0.01)$. Furthermore, the increase in Qmax was also correlated with baseline TPV $(\mathrm{r}=0.31, \mathrm{P}=0.05)$. The data above highlighted TPV as a predictor of outcomes of PAE.

However, Bilhim et al. (21) didn't identify correlation between baseline TPV and clinical failure using Cox regression analysis with hazard ratio of 0.999 (95\% CI: $0.995-1.002 ; \mathrm{P}=0.46)$. In addition to the various criteria in patient selection, different statistical models and, in particular, the specific definitions of clinical failure may explain the discrepancy. Clinical failure in Bilhim's study derived from the concept of clinical success, which was defined as the presence of all of the following: (I) at least a $25 \%$ decrease in IPSS from baseline, (II) an IPSS up to 15 points, (III) at least a 1-point decrease in the QoL score from baseline and a QoL score of up to 3 points, and (IV) no need for any additional medical or surgical therapy for LUTS (25). Obviously, the use of the complex and combined definition of clinical success or failure as variables may lead to conflicting conclusion when comparing to previous analyses by the use of simple variables, e.g., IPSS or Qmax (29,30). De Assis et al. (24) also used a combined parameter of clinical failure with a definition of IPSS $>7$ or QoL $>2$ to correlate baseline TPV with outcomes of PAE, and did not identify statistical correlation between baseline TPV and clinical failure. Interestingly, the authors observed that baseline TPV was significantly correlated to the degree of clinical improvement as assessed by a simple parameter of QoL $(\mathrm{P}<0.001)(24)$.

Other studies also showed contradictory findings. Wang et al. (13) compared the outcomes of PAE between patients with large-volume group (TPV $>80 \mathrm{~mL}$ ) and mediumvolume group (TPV, 50-80 mL). There were no significant differences between groups regarding age, IPSS and Qmax at baseline data. At 12-month follow-up, the authors noted significant differences in the changes of IPSS and Qmax between groups: Large-volume group had a greater reduction in IPSS than the medium-volume group $(\mathrm{P}=0.02)$ and a greater increase in $\mathrm{Qmax}(\mathrm{P}=0.04)$. Likewise, Hacking et al. (46) used ANOVA and $t$ test to analyze 2 groups of patients with TPV $>80 \mathrm{~mL}$ or $\leq 80 \mathrm{~mL}$ and confirmed the relationship of larger TPV with higher IPSS reduction $(\mathrm{P}=0.01)$ but failed to detect significant difference with changes in Qmax. However, in a report by Bagla et al. (12), 78 patients were divided into small-, medium- and largevolume groups with mean baseline TPV of 37.5, 65.7 and $139.4 \mathrm{~mL}$, respectively. ANOVA test between groups on AUA symptom scores at baseline, 1-, 3-, and 6-month data did not identify significant differences at any time points (12), suggesting that baseline TPV is not a predictor for clinical outcomes in PAE. Of note, this study is limited with relatively small sample size in each group. The high dropout rates at 6-month follow-ups are another concern, for example, $47.2 \%(17 / 36)$ in large-volume group and $46.2 \%(12 / 26)$ in medium-volume group, which may potentially lead to biased results.

\section{Central gland/transition zone volume and central gland/transition zone index}

In the normal anatomy of the prostate, the central and transition zones cannot be visualized separately on MR imaging; therefore, radiologists frequently group the two zones under the term of central gland (CG) (47). In patients with $\mathrm{BPH}$, the hyperplasia in the transition zone gradually compresses the central zone into a thin layer of "surgical psudocapsule", which is visible as a faint dark rim on T2 weighted MRI, separating the transition zone from peripheral zone (48). Thus, the size of the central gland is quite close to that of transition zone in elderly patients with BPH. PAE mainly targets at the central gland that represents relatively hypervascular portion of the BPE. Ali et al. (49) observed all infarcts occurred in CG in a prospective clinical trial in 43 patients, subsequently leading to significant shrinkage of the prostate. Furthermore, central gland volume (CGV) reduction was identified at 6-month follow-up in all patients with a median reduction rate of CGV at 26.7\%; while TPV reduction occurred in $93 \%$ of patients with a median reduction rate of TPV at $18.2 \%$ (49). This strongly suggests that CGV or TZV is more responsible to PAE than TPV. Given the strong linear relations between TPV and TZV, which were noted with $\mathrm{r}=0.89$ to $\mathrm{r}=0.95, \mathrm{P}<0.001 \quad(36,38)$ and the significant correlation between baseline TPV with clinical outcomes after PAE as mentioned previously, it is reasonable to speculate that CGV or TZV and CG index 
(CGI: CGV/TPV) or transition zone index (TRI: TRV/ TPV) may correlate with clinical improvement after PAE. This speculation was supported by Abt's post hoc analysis (30), which showed a significant correlation between IPSS changes after PAE and baseline CGV $(\mathrm{r}=-0.34, \mathrm{P}=0.02)$, as well as significant correlations between absolute changes in Qmax and baseline CGV ( $\mathrm{r}=0.39$, $\mathrm{P}=0.01)$ or CGI $(\mathrm{r}=0.46, \mathrm{P}=0.003)$. The modest correlation of IPSS changes and CGV ( $r=-0.34)$ is comparable to that of IPSS changes and TPV $(\mathrm{r}=-0.35)$, whereas the moderate correlation of absolute changes in Qmax and CGI $(r=0.46)$ is stronger than that of the Qmax changes and TPV ( $\mathrm{r}=0.31$ ) (30). In addition, de Assis et al. (24) reported a retrospective study in 93 consecutive patients by the use of QoL at 6 months as a primary endpoint. Although the Spearman's rank correlation coefficients were not available in this report, the authors identified that improvement in QoL had significant correlations with both baseline CGV $(\mathrm{P}<0.001)$ and $\mathrm{CGI}(\mathrm{P}<0.001)$. Furthermore, the cut-off value of baseline CGI for better clinical outcomes was calculated as $>0.45$, with $85 \%$ sensibility and $75 \%$ specificity $(\mathrm{P}<0.05)(24)$.

\section{Prostate volume $(\mathrm{PV})$ reduction}

Although substantial PV reduction usually occur early at 1 month after PAE, a meta-analysis of PAE for BPH with data derived from 19 studies demonstrated that the summary-weighted average PV reduction from baseline PV was $-14.51,-30.11,-28.95$, and $-31.31 \mathrm{~cm}^{3}$ at $1,3,6$, and 12 months, respectively (50). Since the PV reduction at 3 months seems to be double of that at 1 month after PAE, 3-month $\mathrm{PV}$ reduction is an ideal variable in prediction of PAE outcome. In the literature, two statistical methods are commonly used: "outcome variable-A $v s$. outcome variable-B" or "predicting variable-A vs. outcome variable-B". In the former, information of $\mathrm{PV}$ reduction (outcome variable-A) and clinical success/failure or other variable (outcome variable-B) are both collected from the data at the same time point, usually at the end of study. In the latter, the data of $\mathrm{PV}$ reduction (predicting variable-A) are collected earlier, e.g., 3 months after PAE, then those of clinical improvement/failure (outcome variable-B), e.g., at 12-month follow up. The statistical correlation obtained with the former method may suggest a potential of prediction role of PV reduction, whereas the latter directly tests predicting capability of $\mathrm{PV}$ reduction at a certain time point for the consequent clinical improvement.
In a comparative study of medium- and large-volume prostate in PAE, Wang et al. (13) analyzed the data at 12-month follow-up and found that the PV reduction rate had a strong correlation with the clinical improvement $(\mathrm{r}=0.95)$. Although details of statistical analysis for the correlation coefficient were not available, the findings indicated the PV reduction may have potential role in prediction of outcome of PAE. However, Abt et al. (30) observed that the PV reduction did not correlate with relief in IPSS $(\mathrm{r}=0.18, \mathrm{P}=0.23)$, nor with the changes in $\mathrm{Qmax}$ $(\mathrm{r}=-0.18, \mathrm{P}=0.31)$ at 12 weeks after PAE.

By far, there is only one study using the "predicting variable-A vs outcome variable-B" model to test the correlation of PV reduction at 3 months after PAE with the clinical outcome at 12 months (29). In this study, a mean PV reduction at 3 months after PAE was $30.4 \%$. Pearson's correlation test showed that the 3-month PV reduction had a moderate correlation with the changes in IPSS at 12 months $(r=0.68, P<0.001)$. Thus, the coefficient of determination $\left(\mathrm{R}^{2}\right.$, r-squared) can be calculated as 0.462 , indicating that approximately $46 \%$ of the variation in clinical improvement in IPSS at 12 months after PAE can be explained by 3 -month PV reduction. In addition, multiple linear regression test in this study also showed the most significant coefficient was 3-month PV reduction $(\beta=0.61, P<0.001)$.

\section{Prostate median lobe and intravesical prostatic protrusion (IPP)}

In imaging diagnosis of $\mathrm{BPH}$, the enlarged lateral lobe and median lobe represent hyperplastic glands arising from the transition zone and periurethral zone, respectively (48). It is commonly believed that the enlargement of median lobe may result in one or more pedunculated nodules protruding into the urinary bladder, causing mechanical bladder outlet obstruction; although the lateral lobe hyperplasia may also be involved in IPP (51,52). It has been postulated that IPP creates a "ball-valve" type of obstruction, disrupting the funneling effect of the bladder neck, and causing dyskinetic movements of the bladder during voiding (51). IPP is common in aging men and documented with a prevalence of $27 \%$ in healthy Caucasian men between $41-88$ years (53). The measure of IPP or IPP index is defined as the vertical distance from the tip of the intravesical protrusion to the circumference of the bladder at the bladder neck (54). IPP has been widely used as a biomarker in clinical diagnosis 
of BOO, evaluation progression of LUTS, and prediction of treatment outcomes. Urodynamic studies demonstrated that significant IPP in patients with LUTS or BPE was associated with a higher $\mathrm{BOO}$ index (51) and there was a moderate correlation between IPP and BOO index $(r=0.456$, $\mathrm{P}=0.032$ ) (55). In clinical trials on medical therapies, significant IPP has been validated as a reliable predictor for poor responses to $\alpha$-blocker, $5 \alpha$-reductase inhibitors, or combination therapy (56-58).

Recently, IPP has become a new topic of interest in PAE. In a single-center prospective study in 18 patients presented with enlarged median lobe and IPP larger than $5 \mathrm{~mm}$, follow-up at 3 months after PAE showed the mean IPP index decreased significantly together with shrinkage of the enlarged median lobe (54). Furthermore, the authors identified a moderate correlation between the changes in IPP index (\%) and the changes in IPSS (\%) $(r=0.636$, $\mathrm{P}=0.0045)$, suggesting that approximately $40 \%\left(\mathrm{r}^{2}=0.404\right)$ of the variation in clinical relief in IPSS could be explained by the decrease in IPP index (54). In addition, Yu et al. (59), in a prospective study of 82 patients, revealed no significant association between the presence of IPP and suboptimal relief in IPSS and QoL, or suboptimal improvement in Qmax (59). When subclassifying the patients with IPP by a thickness-to-height ratio at a cutoff value (T/H $=1.3$ ), the authors found that IPP with a $\mathrm{T} / \mathrm{H}$ ratio $\leq 1.3$ correlated with suboptimal IPSS at 12 months $(\mathrm{P}=0.025)$ and suboptimal QOL at 6 months $(\mathrm{P}=0.025)$ and 12 months $(\mathrm{P}=0.008)$. Moreover, the patients with IPP tended to have more complications such as de novo AUR requiring bail-out surgery (59). Interestingly, one typical case with IPP (T/H $=1$ ) presented with AUR and underwent TURP at 2 months. The pedunculated IPP lesion showed extensive infarction at 2-week MRI examination and was confirmed with complete necrosis in postoperative histopathological study (59). Although PAE could successfully induce desired necrosis in IPP lesions, deterioration in BOO or even AUR may occur due to the anatomic feature and the unique "ball-valve" effect. This highlights the importance of IPP predictive value, especially in the subclassified IPP parameters, which needs further investigation in clinical practice.

\section{Baseline clinical characteristics}

$\mathrm{PAE}$ is indicated in $\mathrm{BPH}$ patients with moderate to severe LUTS. Most commonly accepted inclusion criteria include patients with IPSS $>8$, Qmax $<12 \mathrm{~mL} / \mathrm{s}$ or presence of urinary retention (5). The baseline parameters, such as IPSS, Qmax, and urinary retention, in prediction of outcome of PAE are of clinical implications. Currently, very few studies focused on analyses of these predictive factors.

\section{Baseline IPSS}

In a multivariate analysis with random-effects linear regression model, Bilhim et al. (21) identified baseline IPSS as an independent predictor of the changes in IPSS at follow-up data $(\mathrm{P}<0.001)$ and stated that patients with lower baseline IPSS, in particular less than 23 points, are ideal candidates for PAE. However, Cox regression analysis for clinical failure didn't support baseline IPSS as an independent predictor $(\mathrm{P}=0.96)$ (21). In addition, $\mathrm{Abt}$ et al. (30) used Spearman rank correlation test and revealed that clinical relief in IPSS after PAE did not correlate with either baseline total IPSS $(\mathrm{P}=0.9)$, IPSS-storage symptoms $(\mathrm{P}=0.38)$, or IPSS-voiding symptoms $(\mathrm{P}=0.25)$. Instead, baseline total IPSS had a modest correlation with increase of Qmax ( $\mathrm{r}=-0.37, \mathrm{P}=0.02)$ after PAE, and baseline IPSSvoiding symptoms correlated with more pronounced improvement in $\mathrm{Qmax}(\mathrm{r}=-0.49, \mathrm{P}=0.001)(30)$.

\section{Baseline Qmax}

After Bilhim et al. (21) initially excluded baseline Qmax as an independent predictor for clinical failure after PAE with a hazard ratio of $0.984(\mathrm{P}=0.38)$ in a univariate analysis of Cox regression model, Abt et al. (30) also confirmed in a Spearman rank correlation test that the baseline Qmax had no significant correlation with improvement of IPSS ( $\mathrm{r}=-0.07, \mathrm{P}=0.62)$ after PAE. However, the authors identified a modest statistically significant correlation $(\mathrm{r}=-0.43, \mathrm{P}=0.005)$ between baseline $\mathrm{Qmax}$ and absolute increase of Qmax at 12 weeks after PAE (30). This suggested that patients with lower Qmax might benefit from PAE with more increase in peak flow rate after treatment.

\section{Urinary retention}

BPH patient with indwelling bladder catheter due to acute urinary retention (AUR) is an ideal indication for PAE. Previous observations showed patients with urinary catheter at the time of procedure accounted for $7.0-18.8 \%$ of all patients $(5,13,30)$. Successful removal of the bladder catheter was reported from $91 \%$ to $100 \%$, which occurred between 2 days and 3 months after PAE $(5,13,30,60)$. The underlying mechanism was explained 
with prostatic inflammation that usually occurs with bladder catheterization, leading to arterial vasodilatation and rendering the prostate gland more susceptible to ischemia (21). Bilhim identified a hazard ratio of 0.486 $(\mathrm{P}=0.027)$ for $\mathrm{PAE}$ clinical failure in a univariate analysis of Cox regression model, suggesting urinary retention as an independent predictor for clinical outcome of PAE (21). Although Abt et al. (30) observed that indwelling bladder catheter might explain a median difference of $14.83 \%$ in relief of IPSS after PAE, the correlation was not of statistical significance $(\mathrm{P}=0.25)$. Nevertheless, indwelling bladder catheter significantly correlated with increase in Qmax after $\mathrm{PAE}$ at a median difference of $12.40 \mathrm{~mL} / \mathrm{s}(\mathrm{P}<0.001)(30)$.

\section{Pathological feature: adenomatous-dominant BPH (AdBPH)}

Adenomatous-dominant BPH was first described by Little et al. (14) as two or more adenomas within the periurethral transition zone of $1 \mathrm{~cm}$ or greater in diameter determined on pre-PAE multi-parametric MRI. Although various $\mathrm{BPH}$ nodules have been identified histologically, including adenofibromatous, fibromuscular, muscular, fibroadenomatous, and stromal nodules, the adenomas in Little's study mainly represented adenofibromatous nodules (14). Previous studies have suggested that the predominant component of the $\mathrm{BPH}$ nodules may dictate the response to therapies: smooth muscle predominant nodules would respond to a-blockers, epithelial nodules to 5 a-reductase inhibitors, and fibrous nodules to surgery (61). In terms of PAE, based on the findings of animal experiments in a canine glandular hyperplasia model, Sun et al. (15) inferred that epithelial nodules in human $\mathrm{BPH}$ might have more response to PAE than other nodules. Little et al. (14) confirmed that all adenomas within the AdBPH group exhibited hyperenhancement post-gadolinium contrast injection compared to the surrounding central gland on multi-parametric MRI. Since hypervascularity of a tumor generally indicates its high demand of oxygen to maintain the higher level of metabolism, the hypervascular lesions are usually more susceptible to ischemia, e.g., the local anoxia induced by PAE. On the other hand, the increased vascularity of the adenomas in the prostate represents the more feeding blood flow compared to the surrounding tissue, so that the preferential flow to the adenomas may carry more embolic particles in embolization procedure and results in significant necrosis or infarction inside the adenomas. These pose the rationale for $\mathrm{AdBPH}$ as a proposed predictor in PAE.

In a report by Little et al. (14), 24 patients were enrolled in AdBPH and Non-AdBPH groups, with 12 patients each. The authors used multivariate ANOVA to compare and analyze the data of IPSS, IIEF, and QoL before and after PAE between the 2 groups, and found a significant reduction in IPSS in the AdBPH group $(\mathrm{P}=0.01)$. QOL scores significantly improved in the $\mathrm{AdBPH}$ group $(\mathrm{P}=0.007)$. Accordingly, AdBPH was for the first time evaluated as clinical predictors for improvement of IPSS and its QOL scores. However, the changes in Qmax were not included as an outcome variable in the study, thus losing an important parameter in evaluation on predicting capability of AdBPH in PAE.

To test the correlation between AdBPH and improvement in Qmax after PAE, Abt et al. (30) enrolled 48 patients and divided into 3 groups with one adenomatous nodule of $\geq 1 \mathrm{~cm}$ diameter ( $\mathrm{n}=13$ ), and more than 2 adenomatous nodules $(n=10)$, and Non-adenomatous nodule $(n=25)$, respectively. By using Spearman rank correlation coefficient, the authors confirmed a modest correlation between AdBPH and absolute change in $\mathrm{Qmax}(\mathrm{r}=0.35, \mathrm{P}=0.02)$. However, statistically significant correlation either between AdBPH $(r=-0.25, P=0.08)$ and relative changes in IPSS or between AdBPH and prostate volume reduction ( $r=-0.07$, $\mathrm{P}=0.64$ ) was not detected (30).

In comparison of the two studies above, the inconsistent findings are attributed to the distinct definitions of AdBPH as an independent variable and various statistical models used. In Little's study (14), AdBPH was defined only in patients with two or more adenomas of $1 \mathrm{~cm}$ or greater in diameter. Analysis with multivariate ANOVA indicated significant reduction in IPSS in patients of the specifically defined AdBPH group compared with those in non-AdBPH group (14). In Abt's statistical model of Spearman rank correlation coefficient (30), ordinal variables (non-AdBPH, AdBPH with one adenoma, and AdBPH with 2 or more adenomas) were collected, thus leading to discrepancy in conclusions. More studies are needed to address the inconsistence.

\section{Prostate tissue ischemia and infarction}

PAE-induced prostate tissue ischemia contributes to a pivotal mechanism in relief of $\mathrm{BOO}$ and associated clinical symptoms by affecting on both the static and dynamic pathological components of BPH (16). On the one hand, the prostate tissue ischemia rapidly leads to ischemic 
necrosis or infarction, evoking the local inflammatory reactions and the subsequent tissue repair, and finally debulking the enlarged prostate in order to achieve the therapeutic purpose. Thus, early detection of prostate tissue ischemia or infarction has been deemed as a potential predictor for future clinical outcomes (21). On the other hand, the prostate tissue ischemia also plays a role under other mechanisms by reducing prostate smooth muscle tone or inducing prostate apoptosis (16). These additional mechanisms may enhance the therapeutic effects when a large prostate infarction occurs after PAE. However, even if no significant prostatic infarction is identified after PAE, some patients still show substantial clinical improvement due to the additional mechanisms. This may explain in part the current discrepancy in evaluation of prostate tissue ischemia or infarction as predictors for clinical outcomes after PAE.

Imaging detection of prostate tissue ischemia or infarction is most commonly used by means of contrastenhanced magnetic resonance imaging (CEMRI) $(21,62,63)$. Recently, contrast-enhanced ultrasonography (CEUS) and surrogate MRI parameters of prostate vascularization in dynamic CEMRI have also been reported $(64,65)$. In CEMRI and CEUS, the prostate tissue ischemia and infarcted areas were defined as new regions that displayed no evident enhancement after contrast agent administration $(62,64)$. Kisilevzky et al. (63) reported PAE in 24 patients with long-term indwelling urinary catheters and observed that 15 patients $(63 \%)$ successfully removed the urinary catheter within 60 days after PAE. All patients underwent the CEMRI 30 days after PAE. The authors identified prostate ischemic areas in 13 out of 15 patients $(87 \%)$ in the group of clinical success, whereas only 1 out of 9 patients $(11 \%)$ in the group of failure showed a small area of ischemia. Mann-Whitney test showed that the presence of ischemia $(\mathrm{P}<0.05)$ and mean percentage of infarcted tissue $(\mathrm{P}=0.0006)$ were significantly different between the two groups. Furthermore, Spearman's rank correlation test suggested a moderately strong correlation $(r=-0.61$, $\mathrm{P}=0.0017$ ) between percentage of tissue ischemia and the time needed to remove the urinary catheter, indicating that MRI assessment of prostatic ischemia at 1 month after PAE is a reliable predictor of clinical success in patients with indwelling urinary catheter. In addition, Bilhim et al. (21) confirmed the predictive role of prostate ischemia in 48 patients. CEMRI was conducted 2-4 weeks after PAE to detect and measure tissue ischemia. By using random effect regression model, the authors found lower IPSS during follow-up was associated with higher percentage of tissue ischemia, suggesting that prostate tissue ischemia correlated with clinical outcome. Although Amouyal et al. (10) also found a significant correlation between infarction and clinical success $(\mathrm{P}=0.002)$ with the test of Spearman correlation coefficient, this study couldn't provide direct evidence on the predictive role of infarction because the data of the presence of infarction and clinical parameters were collected at the same time point, 3 months after PAE.

In contrast to Amouyal's findings, Frenk et al. (62) observed no statistically significant association between the development of infarcts and mean IPSS after PAE. However, the conclusion was based on a specific cohort of patients with clinical success, whereas all patients of clinical failure had been excluded. Therefore, the statistical findings between the 2 groups suggested that IPSS would not correlate with the presence of prostatic infarcts only in patients with clinical success after PAE. Similarly, Lin et al. (66) also got a conclusion that infarction detected at 3 months after PAE had no significant impact on clinical success at the same time. However, no detailed information on either infarction, clinical data, or statistical analysis was provided by the authors.

The timing for detection of the prostatic ischemia or infarction that may potentially predict clinical outcomes after PAE has recently gained interest. Previous studies commonly assessed prostatic ischemia as a Type 3 predictor, the data of which were collected at one month or later after PAE and used for long-term clinical outcomes. From clinical perspective, earlier prediction for clinical prognosis is of more importance in order to modify treatment plan in time in those patients with likely clinical failure after PAE. Therefore, prostatic ischemia has been suggested as Type II instead of Type III predictor, so that MRI examinations to detect and measure prostatic ischemia would be performed at first 24 hours or between 1-2 weeks (21). Unfortunately, current evidence on prostate ischemia at 24 hours after PAE did not support its predictive role in clinical practice $(64,65)$. While further addressing the predictive role of ischemia at 1-day after PAE in more patients, we suggest more future studies focusing on prostate ischemia at 1 week after PAE in prediction of the medium-term or long-term clinical outcomes.

\section{PSA/C-reactive protein}

Prostate-specific antigen, one of the major components 
of protein in the prostate fluid, is a glycoprotein enzyme secreted by the glandular epithelium. Men normally have low PSA levels in serum. When the epithelial cells, basement membrane of the gland, and prostate stroma are damaged and the vascular permeability is increased due to a variety of pathologies, such as prostate cancer, $\mathrm{BPH}$, and prostatitis; PSA releases into blood stream, resulting in its elevated blood levels. In medical therapy with 5-alpha reductase inhibitors, baseline PSA as well as prostate volume has been validated as a good predictor of long-term relief in LUTS and improvement in urinary flow rate (67). However, evaluation of the predictive value of baseline PSA in PAE is currently scarcely reported. Bilhim et al. (21) used a univariate analysis of Cox regression model to test baseline PSA in prediction of clinical failure after PAE and found a no significant correlation with a hazard ratio of 0.972 $(\mathrm{P}=0.10)$. In a post-hoc analysis of 48 patients undergone PAE, Abt et al. (30) didn't to reveal statistically significant relationships among either baseline PSA and relative change in IPSS ( $\mathrm{r}=-0.19)$ or baseline PSA and absolute change in $\mathrm{Qmax}(\mathrm{r}=0.21)$.

In contrast to baseline PSA, the PSA level at 24 hours after PAE seems to have a potential in early prediction of clinical outcomes. PAE-induced acute focal prostate necrosis may result in an acute release of a large amount PSA into systemic circulation and a peak serum PSA level at 24-48 hours. Wang et al. found the mean serum PSA level at 24 hours after PAE increased up to 24.4 folds compared to the baseline data (13); likewise, the 24-hour post-PAE PSA level had a significant strong correlation with ischemia volume detected with MRI at 1 week after PAE (Spearman correlation coefficient $r=0.87 ; \mathrm{P}=0.017$ ) (68). Since the correlation between ischemia volume after PAE and the subsequent clinical outcomes has been validated $(21,63,68)$, the hypothesis of the predictive role of the serum level of PSA at 24 hours after PAE is plausible. In a prospective single-center study of PAE in 35 patients with prostate larger than $90 \mathrm{~g}$, de Assis et al. (69) first identified the serum PSA level at 24 hours after PAE was moderately correlated with relief in IPSS at 3-month follow-up by the use of Spearman correlation coefficient test $(\mathrm{r}=0.4773 ; \mathrm{P}=0.0057)$. Subsequently, Bilhim et al. (21) evaluated the predictive role of the 24-hour post-PAE PSA level in 133 patients and confirmed the significant association between higher levels of PSA at 24 hours after PAE and lower IPSS over time $(\mathrm{P}=0.01)$. Moreover, the authors noticed that patients with at least of $75 \mathrm{ng} / \mathrm{mL}$ had a greater decrease in IPSS than those with less than $75 \mathrm{ng} / \mathrm{mL}(\mathrm{P}=0.01)$. In addition, Wang et al. (70) compared the 24-hour post-PAE PSA levels between patients with and without clinical success and found that patients with clinical success had a mean 20.7-fold increase in 24-hour post-PAE PSA level whereas patients with clinical failure had a mean 7.0-fold increase in PSA level at 24 hours $(\mathrm{P}<0.01)$, supporting the hypothesis that the 24-hour post-PAE PSA level has a significant relationship with clinical success or failure. However, Abt et al. (30), with Spearman rank correlation analysis in 48 patients, failed to identify statistically significant correlations between either PSA level at 24-48 hours and relative changes in IPSS at 12 weeks after PAE $(\mathrm{P}=0.48$, $\mathrm{r}=-0.10$ ) or absolute changes in Qmax 12 weeks after PAE $(\mathrm{P}=0.44, \mathrm{r}=0.12)$. Instead, the authors noticed a modest correlation between C-reactive protein (CRP) level at 2448 hours and relative changes in IPSS at 12 weeks after PAE $(\mathrm{r}=-0.30, \mathrm{P}=0.04)$. Therefore, CRP at 48 hours after PAE might be a potential early predictor. More clinical trials are needed to address its predictive value in PAE for its clinical outcomes.

\section{Conclusions}

Studies on clinical predictors in PAE remain in early stage. The 3-type classification of the potential clinical predictors in PAE may help clarify and group various clinical parameters in clinical evaluation. Although various clinical reports on PAE predictive factors have gained increasing interest, conflicting findings and even contradictory results are commonly seen (Table 3). The reasons for inconsistency are multifactorial, including the diverse sample size of patients, different patients' selection criteria, absence of the standard parameters in clinical outcomes (clinical success or clinical failure), and inappropriate study design or statistical analysis. Taken together, current evidence suggests baseline prostate volume, in particular TZV and TZI, $24 \mathrm{~h}$ postPAE PSA level, and prostate infarction and prostate volume reduction have potential in prediction of treatment outcomes. Patients with Adenomatous-dominant BPH or with indwelling bladder catheter before PAE may have more benefits from PAE. Baseline IPP, CRP level at 48 hours and early detection of prostate infarct at 1 day and 1 week after PAE need further investigating. More prospective clinical trials with large sample size of patients are mandatory. 
Table 3 Summary of the major predictor in PAE

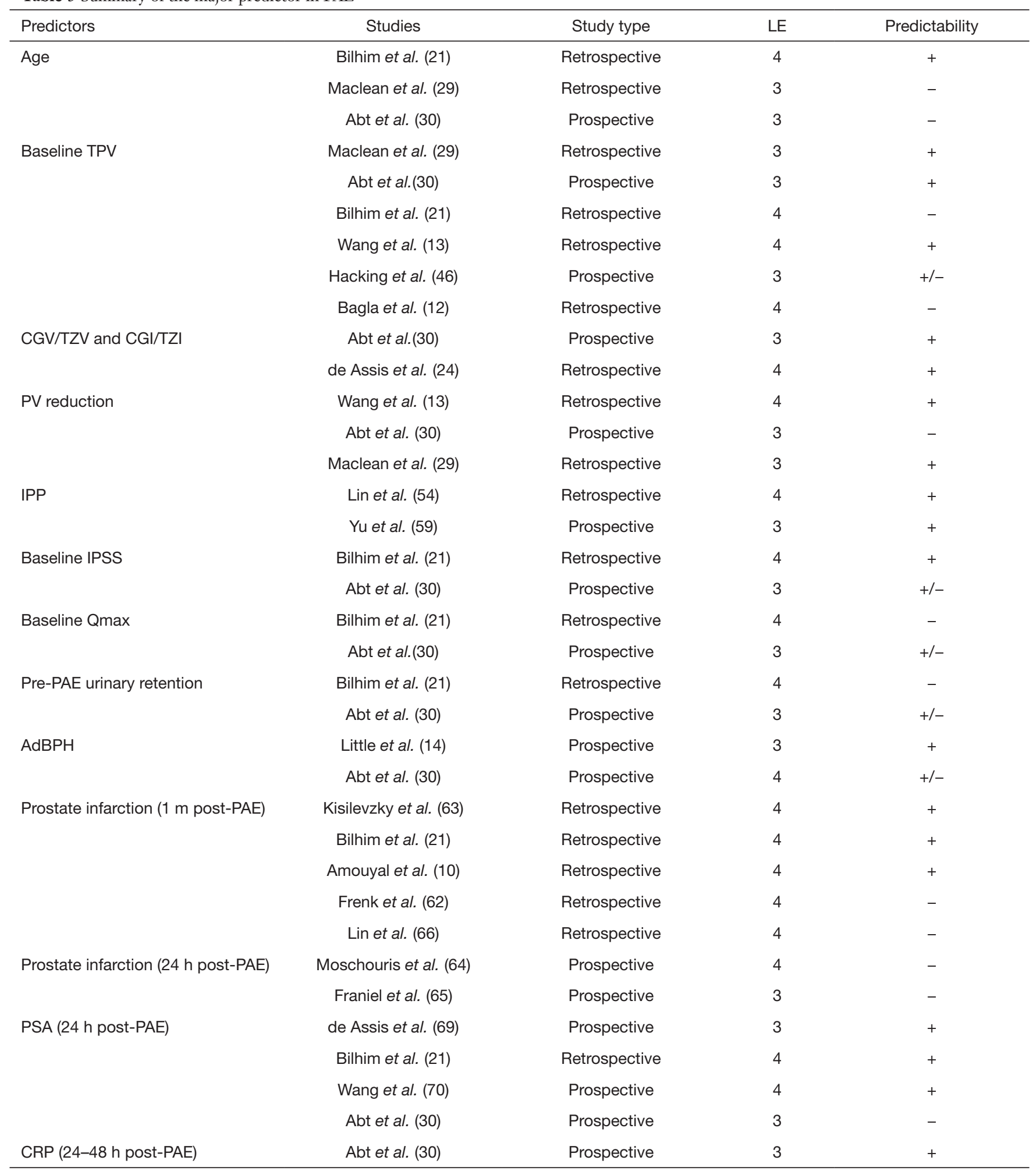

LE, level of evidence (Oxford 2011 Levels of Evidence using the protocol for treatment benefits studies, Oxford Centre for Evidence-Based Medicine. http://www.cebm.net/index.aspx?o=5653). Predictability (+)/Predictability (-): with/without statistical significance in prediction of a particular outcome parameter. Predictability $(+/-)$ : inconsistent results in prediction of different outcome parameters, e.g., changes in IPSS and Qmax. 


\section{Acknowledgments}

Funding: This study was sponsored in part by grant IB18129 from Plan Regional de Investigación, Consejería de Economía, Ciencia y Agenda Digital, Junta de Extremadura and Fondo Europeo de Desarrollo Regional.

\section{Footnote}

Conflicts of Interest: All authors have completed the ICMJE uniform disclosure form (available at http://dx.doi. org/10.21037/tau-20-437). The authors have no conflicts of interest to declare.

Ethical Statement: The authors are accountable for all aspects of the work in ensuring that questions related to the accuracy or integrity of any part of the work are appropriately investigated and resolved. This study did not require approval by the institutional review board at Jesus Uson Minimally Invasive Surgery Centre, Caceres, Spain.

Open Access Statement: This is an Open Access article distributed in accordance with the Creative Commons Attribution-NonCommercial-NoDerivs 4.0 International License (CC BY-NC-ND 4.0), which permits the noncommercial replication and distribution of the article with the strict proviso that no changes or edits are made and the original work is properly cited (including links to both the formal publication through the relevant DOI and the license). See: https://creativecommons.org/licenses/by-nc-nd/4.0/.

\section{References}

1. Sun F, Sánchez FM, Crisóstomo V, et al. Benign prostatic hyperplasia: Transcatheter arterial embolization as potential treatment - Preliminary study in pigs. Radiology 2008;246:783-9.

2. Mauro MA. Science to practice: Can hyperplastic prostate follow uterine fibroids and be managed with transcatheter arterial embolization? Radiology 2008;246:657-8.

3. Carnevale FC, Antunes AA, Da Motta Leal Filho JM, et al. Prostatic artery embolization as a primary treatment for benign prostatic hyperplasia: Preliminary results in two patients. Cardiovasc Intervent Radiol 2010;33:355-61.

4. Pisco JM, Pinheiro LC, Bilhim T, et al. Prostatic arterial embolization to treat benign prostatic hyperplasia. J Vasc Interv Radiol 2011;22:11-9.

5. Pisco J, Pinheiro LC, Bilhim T, et al. Prostatic arterial embolization for benign prostatic hyperplasia: Short-And intermediate-term results. Radiology 2013;266:668-77.

6. Pisco JM, Bilhim T, Pinheiro LC, et al. Medium- and Long-Term Outcome of Prostate Artery Embolization for Patients with Benign Prostatic Hyperplasia: Results in 630 Patients. J Vasc Interv Radiol 2016;27:1115-22.

7. Bilhim T, Pisco J, Campos Pinheiro L, et al. Does polyvinyl alcohol particle size change the outcome of prostatic arterial embolization for benign prostatic hyperplasia? Results from a single-center randomized prospective study. J Vasc Interv Radiol 2013;24:1595602.e1.

8. Gonçalves OM, Carnevale FC, Moreira AM, et al. Comparative Study Using 100-300 Versus 300-500 $\mu \mathrm{m}$ Microspheres for Symptomatic Patients Due to Enlarged-BPH Prostates. Cardiovasc Intervent Radiol 2016;39:1372-8.

9. Carnevale FC, Moreira AM, Antunes AA. The "PErFecTED Technique": Proximal Embolization First, Then Embolize Distal for Benign Prostatic Hyperplasia. Cardiovasc Intervent Radiol 2014;37:1602-5.

10. Amouyal G, Thiounn N, Pellerin O, et al. Clinical Results After Prostatic Artery Embolization Using the PErFecTED Technique: A Single-Center Study. Cardiovasc Intervent Radiol 2016;39:367-75.

11. Bagla S, Rholl KS, Sterling KM, et al. Utility of conebeam CT imaging in prostatic artery embolization. J Vasc Interv Radiol 2013;24:1603-7.

12. Bagla S, Smirniotopoulos JB, Orlando JC, et al. Comparative analysis of prostate volume as a predictor of outcome in prostate artery embolization. J Vasc Interv Radiol 2015;26:1832-8.

13. Wang M, Guo L, Duan F, et al. Prostatic arterial embolization for the treatment of lower urinary tract symptoms caused by benign prostatic hyperplasia: A comparative study of medium- and large-volume prostates. BJU Int 2016;117:155-64.

14. Little MW, Boardman P, Macdonald AC, et al. Adenomatous-Dominant Benign Prostatic Hyperplasia (AdBPH) as a Predictor for Clinical Success Following Prostate Artery Embolization: An Age-Matched CaseControl Study. Cardiovasc Intervent Radiol 2017;40:682-9.

15. Sun F, Crisóstomo V, Báez-Díaz C, et al. Prostatic Artery Embolization (PAE) for Symptomatic Benign Prostatic Hyperplasia (BPH): Part 1, Pathological Background and Clinical Implications. Cardiovasc Intervent Radiol 2016;39:1-7. 
16. Sun F, Crisóstomo V, Báez-Díaz C, et al. Prostatic Artery Embolization (PAE) for Symptomatic Benign Prostatic Hyperplasia (BPH): Part 2, Insights into the Technical Rationale. Cardiovasc Intervent Radiol 2016;39:161-9.

17. McWilliams JP, Bilhim TA, Carnevale FC, et al. Society of Interventional Radiology Multisociety Consensus Position Statement on Prostatic Artery Embolization for Treatment of Lower Urinary Tract Symptoms Attributed to Benign Prostatic Hyperplasia: From the Society of Interventional Radiology, the Cardiovascular and Interventional Radiological Society of Europe, Société Française de Radiologie, and the British Society of Interventional Radiology: Endorsed by the Asia Pacific Society of Cardiovascular and Interventional Radiology, Canadian Association for Interventional Radiology, Chinese College of Interventionalists, Interventional Radiology Society of Australasia, Japanese Society of Interventional Radiology, and Korean Society of Interventional Radiology. J Vasc Interv Radiol 2019;30:627-37.e1.

18. NICE Guidance - Prostate Artery Embolisation for Lower Urinary Tract Symptoms Caused by Benign Prostatic Hyperplasia: (C NICE (2018) Prostate Artery Embolisation for Lower Urinary Tract Symptoms Caused by Benign Prostatic Hyperplasia. BJU Int 2018;122:11-2.

19. Gidron Y. Clinical Predictors. In: Gellman MD, Turner JR (eds). Encyclopedia of Behavioral Medicine. New York: Springer, 2013; 427-8.

20. Han K, Song K, Choi BW. How to develop, validate, and compare clinical prediction models involving radiological parameters: Study design and statistical methods. Korean J Radiol 2016;17:339-50.

21. Bilhim T, Pisco J, Pereira JA, et al. Predictors of clinical outcome after prostate artery embolization with spherical and nonspherical polyvinyl alcohol particles in patients with benign prostatic hyperplasia. Radiology 2016;281:289-300.

22. Sagnier PP, MacFarlane G, Teillac P, et al. Impact of Symptoms of Prostatism on Level of Bother and Quality of Life of Men in the French Community. J Urol 1995;153:669-73.

23. Wang MQ, Wang Y, Yan JY, et al. Prostatic artery embolization for the treatment of symptomatic benign prostatic hyperplasia in men $\geq 75$ years: a prospective single-center study. World J Urol 2016;34:1275-83.

24. de Assis AM, Maciel MS, Moreira AM, et al. Prostate Zonal Volumetry as a Predictor of Clinical Outcomes for Prostate Artery Embolization. Cardiovasc Intervent Radiol 2017;40:245-51.
25. Grosso M, Balderi A, Arnò M, et al. Prostatic artery embolization in benign prostatic hyperplasia: preliminary results in 13 patients. Radiol Med 2015;120:361-8.

26. Khalili M, Lam W, Challacombe B, et al. Successful Treatment of Benign Prostatic Hyperplasia Using Prostate Artery Embolization in a 97-Year-Old Patient Presenting With Urinary Retention and Visible Haematuria. Int J Surg 2017;47:S25.

27. Shahar E, Folsom AR, Romm FJ, et al. Patterns of aspirin use in middle-aged adults: The atherosclerosis risk in communities (ARIC) study. Am Heart J 1996;131:915-22.

28. Fusco F, Arcaniolo D, Creta M, et al. Demographic and comorbidity profile of patients with lower urinary tract symptoms suggestive of benign prostatic hyperplasia in a real-life clinical setting: Are 5-alpha-reductase inhibitor consumers different? World J Urol 2015;33:685-9.

29. Maclean D, Harris M, Drake T, et al. Factors Predicting a Good Symptomatic Outcome After Prostate Artery Embolisation (PAE). Cardiovasc Intervent Radiol 2018;41:1152-9.

30. Abt D, Müllhaupt G, Mordasini L, et al. Outcome prediction of prostatic artery embolization: post hoc analysis of a randomized, open-label, non-inferiority trial. BJU Int 2019;124:134-44.

31. Kaplan SA. Re: Prostatic Arterial Embolization to Treat Benign Prostatic Hyperplasia. J Urol 2012;187:212-3.

32. McVary KT. Prostatic arterial embolization for LUTS/ $\mathrm{BPH}$ - An opportunity for collaborative research or next prostatic gizmo candidate? J Urol 2014;191:577-8.

33. Barry MJ, Cockett ATK, Holtgrewe HL, et al. Relationship of symptoms of prostatism to commonly used physiological and anatomical measures of the severity of benign prostatic hyperplasia. J Urol 1993;150:351-8.

34. Bosch JLHR, Kranse R, van Mastrigt R, et al. Reasons for the Weak Correlation Between Prostate Volume and Urethral Resistance Parameters in Patients With Prostatism. J Urol 1995;153:689-93.

35. Girman CJ, Jacobsen SJ, Guess HA, et al. Natural History of Prostatism: Relationship Among Symptoms, Prostate Volume and Peak Urinary Flow Rate. J Urol 1995;153:1510-5.

36. Kaplan SA, Te AE, Pressler LB, et al. Transition Zone Index as a Method of Assessing Benign Prostatic Hyperplasia: Correlation with Symptoms, Urine Flow and Detrusor Pressure. J Urol 1995;154:1764-9.

37. Ezz el Din K, Kiemeney LALM, De Wildt MJAM, et al. Correlation between uroflowmetry, prostate volume, postvoid residue, and lower urinary tract symptoms as 
measured by the international prostate symptom score. Urology 1996;48:393-7.

38. Lepor H, Nieder A, Feser J, et al. Total prostate and transition zone volumes, and transition zone index are poorly correlated with objective measures of clinical benign prostatic hyperplasia. J Urol 1997;158:85-8.

39. Øverland GB, Vatten L, Rhodes T, et al. Lower urinary tract symptoms, prostate volume and uroflow in Norwegian community men. Eur Urol 2001;39:36-41.

40. Agrawal CS, Chalise PR, Bhandari BB. Correlation of prostate volume with international prostate symptom score and quality of life in men with benign prostatic hyperplasia. Nepal Med Coll J 2008;10:104-7.

41. Guneyli S, Ward E, Peng Y, et al. MRI evaluation of benign prostatic hyperplasia: Correlation with international prostate symptom score. J Magn Reson Imaging 2017;45:917-25.

42. Lepor H, Machi G. Comparison of aua symptom index in unselected males and females between fifty-five and seventy-nine years of age. Urology 1993;42:36-40.

43. Roehrborn CG. Benign prostatic hyperplasia: etiology, pathophysiology, epidemiology, and natural history. In: Wein AJ, Kavoussi LR, Novick AC, et al. editors. Campbell-Walsh Urology. 10th ed. Philadelphia: Saunders, 2012; 2570-610.

44. Carnevale FC, Moreira AM, Harward SH, et al. Recurrence of Lower Urinary Tract Symptoms Following Prostate Artery Embolization for Benign Hyperplasia: Single Center Experience Comparing Two Techniques. Cardiovasc Intervent Radiol 2017;40:366-74.

45. Bilhim T, Pisco JM, Rio Tinto H, et al. Prostatic arterial supply: Anatomic and imaging findings relevant for selective arterial embolization. J Vasc Interv Radiol 2012;23:1403-15.

46. Hacking N, Vigneswaran G, Maclean D, et al. Technical and Imaging Outcomes from the UK Registry of Prostate Artery Embolization (UK-ROPE) Study: Focusing on Predictors of Clinical Success. Cardiovasc Intervent Radiol 2019;42:666-76.

47. Vargas HA, Akin O, Franiel T, et al. Normal central zone of the prostate and central zone involvement by prostate cancer: Clinical and $\mathrm{mr}$ imaging implications. Radiology 2012;262:894-902.

48. Villeirs GM, Verstraete KL, De Neve WJ, et al. Magnetic resonance imaging anatomy of the prostate and periprostatic area: A guide for radiotherapists. Radiother Oncol 2005;76:99-106.

49. Ali R, Gabr A, Mouli SK, et al. MR imaging findings of the prostate gland following prostate artery embolization: results from a prospective phase 2 study. Abdom Radiol (NY) 2019;44:713-22.

50. Uflacker A, Haskal ZJ, Bilhim T, et al. Meta-Analysis of Prostatic Artery Embolization for Benign Prostatic Hyperplasia. J Vasc Interv Radiol 2016;27:1686-97.e8.

51. Chia SJ, Heng CT, Chan SP, et al. Correlation of intravesical prostatic protrusion with bladder outlet obstruction. BJU Int 2003;91:371-4.

52. Wasserman NF, Spilseth B, Golzarian J, et al. Use of mri for lobar classification of benign prostatic hyperplasia: Potential phenotypic biomarkers for research on treatment strategies. AJR Am J Roentgenol 2015;205:564-71.

53. Audouin M, Girshovich A, Cussenot O, et al. Typology of intravesical prostatic protrusions, or so-called median lobes, in middle-aged and older men. Surg Radiol Anat 2018;40:389-93.

54. Lin YT, Amouyal G, Thiounn N, et al. Intra-vesical Prostatic Protrusion (IPP) Can Be Reduced by Prostatic Artery Embolization. Cardiovasc Intervent Radiol 2016;39:690-5.

55. Keqin Z, Zhishun X, Jing Z, et al. Clinical Significance of Intravesical Prostatic Protrusion in Patients with Benign Prostatic Enlargement. Urology 2007;70:1096-9.

56. Cumpanas AA, Botoca M, Minciu R, et al. Intravesical prostatic protrusion can be a predicting factor for the treatment outcome in patients with lower urinary tract symptoms due to benign prostatic obstruction treated with tamsulosin. Urology 2013;81:859-63.

57. Hirayama K, Masui K, Hamada A, et al. Evaluation of Intravesical Prostatic Protrusion as a Predictor of Dutasteride-Resistant Lower Urinary Tract Symptoms/ Benign Prostatic Enlargement with a High Likelihood of Surgical Intervention. Urology 2015;86:565-9.

58. Yoshida T, Kinoshita H, Yoshida K, et al. Intravesical Prostatic Protrusion as a Predicting Factor for the Adverse Clinical Outcome in Patients with Symptomatic Benign Prostatic Enlargement Treated with Dutasteride. Urology 2016;91:154-7.

59. Yu SCH, Cho CCM, Hung EHY, et al. Thickness-toHeight Ratio of Intravesical Prostatic Protrusion Predicts the Clinical Outcome and Morbidity of Prostatic Artery Embolization for Benign Prostatic Hyperplasia. J Vasc Interv Radiol 2019;30:1807-16.

60. Antunes AA, Carnevale FC, Da Motta Leal Filho JM, et al. Clinical, laboratorial, and urodynamic findings of prostatic artery embolization for the treatment of urinary retention related to benign prostatic hyperplasia. A prospective 
single-center pilot study. Cardiovasc Intervent Radiol 2013;36:978-86.

61. Bostwick DG. The pathology of benign prostatic hyperplasia. In: Kirby RS, McConnell JD, Fitzpatrick JM, et al. editors. Textbook of benign prostatic hyperplasia. 2nd ed. Oxfordshire: Taylor \& Francis, 2005; 97-111.

62. Frenk NE, Baroni RH, Carnevale FC, et al. MRI findings after prostatic artery embolization for treatment of benign hyperplasia. AJR Am J Roentgenol 2014;203:813-21.

63. Kisilevzky N, Faintuch S. MRI assessment of prostatic ischaemia: best predictor of clinical success after prostatic artery embolisation for benign prostatic hyperplasia. Clin Radiol 2016;71:876-82.

64. Moschouris H, Stamatiou K, Malagari K, et al. The value of contrast-enhanced ultrasonography in detection of prostatic infarction after prostatic artery embolization for the treatment of symptomatic benign prostatic hyperplasia. Diagn Interv Radiol 2019;25:134-43.

65. Franiel T, Aschenbach R, Trupp S, et al. Prostatic Artery Embolization with 250- $\mu \mathrm{m}$ Spherical PolyzeneCoated Hydrogel Microspheres for Lower Urinary Tract Symptoms with Follow-up MR Imaging. J Vasc Interv Radiol 2018;29:1127-37.

66. Lin YT, Amouyal G, Correas JM, et al. Can prostatic

Cite this article as: Sun F, Lucas-Cava V, Sánchez-Margallo FM. Clinical predictive factors in prostatic artery embolization for symptomatic benign prostatic hyperplasia: a comprehensive review. Transl Androl Urol 2020;9(4):1754-1768. doi: 10.21037/ tau-20-437 arterial embolisation (PAE) reduce the volume of the peripheral zone? MRI evaluation of zonal anatomy and infarction after PAE. Eur Radiol 2016; 26:3466-73.

67. Roehrborn CG, Boyle P, Bergner D, et al. Serum prostatespecific antigen and prostate volume predict long-term changes in symptoms and flow rate: Results of a four-year, randomized trial comparing finasteride versus placebo. Urology 1999;54:662-9.

68. Wang MQ, Zhang JL, Xin HN, et al. Comparison of Clinical Outcomes of Prostatic Artery Embolization with

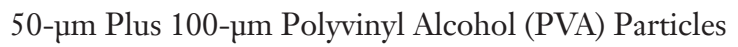
versus 100- $\mathrm{mm}$ PVA Particles Alone: A Prospective Randomized Trial. J Vasc Interv Radiol 2018;29:1694-702.

69. de Assis AM, Moreira AM, De Paula Rodrigues VC, et al. Prostatic artery embolization for treatment of benign prostatic hyperplasia in patients with prostates $>90 \mathrm{~g}$ : A prospective single-center study. J Vasc Interv Radiol 2015;26:87-93.

70. Wang M, Guo L, Duan F, et al. Prostatic arterial embolization for the treatment of lower urinary tract symptoms as a result of large benign prostatic hyperplasia: A prospective single-center investigation. Int J Urol 2015;22:766-72. 